\title{
Informed choice in family planning: What do women want to know?
}

\author{
Cristina López-del Burgo ${ }^{\mathrm{a}, \mathrm{b}}$
}

\section{Alfonso Osorio $^{\mathrm{a}, \mathrm{c}}$}

Jokin de Irala ${ }^{\mathrm{a}, \mathrm{b}}$

${ }^{a}$ Institute for Culture and Society (ICS), University of Navarra, Spain

${ }^{b}$ Department of Preventive Medicine and Public Health, School of Medicine, University of Navarra, Spain

${ }^{c}$ School of Education and Psychology, University of Navarra, Spain

*Correspondence author:

Cristina López-del Burgo

Address: School of Medicine. Irunlarrea 1, 31008 Pamplona, Navarra. SPAIN

Tel: +34948425600 , ext 826360

E-mail address: cldelburgo@unav.es

\section{Published in: \\ Contraception \\ http://www.sciencedirect.com/science/journal/00107824}

See original article in the Journal's web page:

http://www.sciencedirect.com/science/article/pii/S0010782414006489\#

Cite as:

López del Burgo C, Osorio A, de Irala J. Informed choice in family planning: what do women want to know? Contraception 2015; 91(3), 264-265. 
We read with interest the paper from Donnelly et al. about patients' and providers' information priorities for contraceptive decision making [1]. Although the study was conducted in a convenience sample, it highlights the importance of patient-centered contraceptive counseling because providers and women have different priorities sometimes.

We would like to add comments concerning some issues addressed in their discussion. In Donelly's study, having information about mechanisms of action of family planning (FP) methods was ranked in the top 3 priorities for women. We can confirm this as we showed that mechanisms of action do influence women's choice [2]. But we also found low or inaccurate knowledge about mechanisms of action of FP methods, especially among less educated women. The majority of the surveyed women, from 5 European countries, identified the unequivocal mode of action of condoms, sterilization and abortion. However, fewer than $2 \%$ identified all possible pre and postfertilization mechanisms of action of hormonal contraceptives and intrauterine devices [3]. The gap in women's knowledge could be also due to a lack of knowledge of physicians and providers about mechanisms of action of some methods. For example, $25 \%$ of family medicine providers in a Midwestern department from the USA, were unsure about the mechanism of action of the emergency contraception pill [4]. Another possible explanation for women's lack of knowledge could be a poor provider-woman communication. As women believe that physicians and providers are one of the more reliable sources of information about FP, it is important that proffesionals be aware of the best available medical evidence concerning mechanisms of action and that they thoroughly inform women. Poor information about mechanism of action may jeopardize a truly informed choice [5]. 
It would have been interesting for Donelly et al. to explore women's and provider's information priorities taking into account their sociodemographic characteristics and reproductive history. Those findings might help to identify specific needs of women and to improve the contraceptive decision making. For example, according to Donelly's study, how quickly fertility returns after stopping the use of a method was not an information priority for women and providers, but it might be very important for nulliparous women. If so, it becomes relevant to discuss this aspect with them.

We are aware that the first step to improve FP counseling is to identify what women would like to know about FP methods, taking into account their personal situation. Next steps could encompass multiple actions such as improving medical education on FP, teaching women and men about fertility to involve them in their management, evaluating the information given by the providers to women or improving providers' communication skills in FP. But being aware of, and informing clients about available scientific evidence on birth control methods, is essential to assure a truly informed choice in FP.

\section{References}

[1] Donnelly KZ, Foster TC, Thompson R. What matters most? The content and concordance of patients' and providers' information priorities for contraceptive decision making. Contraception 2014 (in press).

[2] Lopez-del Burgo C, Mikolajczyk RT, Osorio A, Errasti T, de Irala J. Women's attitudes towards mechanisms of action of birth control methods: a cross-sectional study in five European countries. J Clin Nurs 2013;22:3006-15.

[3] Lopez-del Burgo C, Mikolajczyk RT, Osorio A, Carlos S, Errasti T, de Irala J. Knowledge and beliefs about mechanism of action of birth control methods among European women. Contraception 2012;85:69-77. 
[4] Wallace JL, Wu J, Weinstein J, Gorenflo DW, Fetters MD. Emergency contraception: knowledge and attitudes of family medicine providers. Fam Med 2004;36:417-22.

[5] Larimore WL, Stanford JB. Postfertilization effects of oral contraceptives and their relationship to informed consent. Arch Fam Med 2000;9:126-33. 